\title{
Two-Dimensional Steady Flow Modeling of Ideal Fluid in Porous Medium Using Finite Element Method
}

\author{
Hairil Anwar ${ }^{*}$, Wahyu Srigutomo ${ }^{2}$ \\ ${ }^{I}$ Magister Program in Informatics, Insitut Teknologi Bandung, Indonesia \\ ${ }^{2}$ Physics of Earth and Complex System Research Division, Insitut Teknologi Bandung, Indonesia
}

Received: 22 May 2015, Revised: 15 July 2015, Accepted: 21 July 2015

\begin{abstract}
Ideal fluid is a fluid which is uncompressed and has no viscosity. A steady stream of ideal fluid in a porous medium can be modeled using finite element method. The finite element method is a numerical method that can be used to solve boundary-value problem governed by a differential equation and a set of boundary conditions. In this modeling, the linear system of equations derived using Galerkin approach for linear triangular elements. Irregular geometry and variation in permeability distribution models are used. The solution obtained in form of fluid head and fluid flow velocity distribution in the modeling domain.
\end{abstract}

Keywords: Finite Element Method, Galerkin, Ideal fluid, Permeability, Steady flow

\section{INTRODUCTION}

Fluid dynamics in porous medium has been investigated by Darcy (1856) and the parameters that related such as permeability of a porous medium [1]. Equation of ideal fluid flow in a steady state has been derived in form of a second order differential equation [2-3]. The problems that arise from the ideal fluid flow could be boundary-value problem which the solution can be obtained numerically by using finite element method [4-5]. The maturity of technology and computation tools made completion of numerical solution more reliable, especially in complex cases where the analytical solution is hard to obtain.

In this paper, we solve two-dimensional (2D) boundary-value problem of steady flow for ideal fluid in porous medium numerically using finite element method. The model used is variation of medium permeability distribution on the domain modeling In order to investigate the effect of permeability distribution on fluid flow through porous medium. We use MATLAB ${ }^{\mathrm{TM}} 2013$ to

Corresponding author.

E-mail address: 23514034@std.stei.itb.ac.id implement the computation of finite element method.

\section{THEORY}

Ideal fluid is a fluid which is uncompressed and has no viscosity. In a steady state, the flow behavior of ideal fluid satisfy following equation:

$$
\nabla \cdot \vec{u}=0
$$

where $\boldsymbol{u}$ is the ideal fluid flow velocity $[\mathrm{m} / \mathrm{s}]$. Equation (1) shows that the volume flow rate of fluid that entering a volume control same as the volume flow rate that leaving the volume control if no source present in the volume control.

Darcy has formulated the relationship between the fluid flow velocity and hydraulic gradient in porous medium. The relationship between the fluid flow velocity and hydraulic gradient is written by the following equation:

$$
\vec{u}=-K \vec{i}=-K \vec{\nabla} \phi
$$


where $\boldsymbol{u}$ is fluid flow velocity [m/s] or also known as Darcy velocity, $K$ is the hydraulic conductivity of porous medium $[\mathrm{m} / \mathrm{s}]$. The hydraulic conductivity of porous medium is calculated by using the following equation:

$$
K=\frac{k \rho_{w} g}{\mu}
$$

where $k$ is hydraulic permeability of porous medium $\left[\mathrm{m}^{2}\right], \rho_{w}$ is fluid density $\left[\mathrm{kg} / \mathrm{m}^{3}\right], g$ is gravity constant $\left[\mathrm{m} / \mathrm{s}^{2}\right]$, and $\mu$ is fluid viscosity [Pa.s]. In this paper, we assume $\rho_{w}$ and $\mu$ are constant so $K$ is proportional to $k$, therefore $K$ can also be referred as permeability. $\boldsymbol{i}$ is hydraulic gradient, and $\varphi$ is fluid head $[\mathrm{m}]$.

The formulation of steady ideal fluid flow in a porous medium obtained by subtituting equation (2) to equation (1):

$$
\vec{\nabla} \cdot(K \vec{\nabla} \phi)=0 .
$$

For two-dimensional steady flow problem, the equation (4) above can be written as:

$$
\frac{\partial}{\partial x}\left(K \frac{\partial \phi}{\partial x}\right)+\frac{\partial}{\partial y}\left(K \frac{\partial \phi}{\partial y}\right)=0
$$

with the assumption that permeability of the porous medium in $\mathrm{x}$ direction is equal to it's permeability in $y$ direction. The variables used in equation (4) and equation (5) also the variables used in equation (2).

\section{EXPERIMENTAL METHOD}

Two-dimensional steady flow in a porous medium is a boundary value problem that can be solved by using numerical methods such as finite difference method and finite element method. The method used in this modeling is the finite element method with Galerkin approach for linear triangular element [5]. In finite element method, the numerical procedures consist of domain discretization into triangle elements, element permeability value assignment, assembly of global matrix system of equations, imposing the boundary conditions, and the calculation of a solution of fluid head and fluid flow velocity.

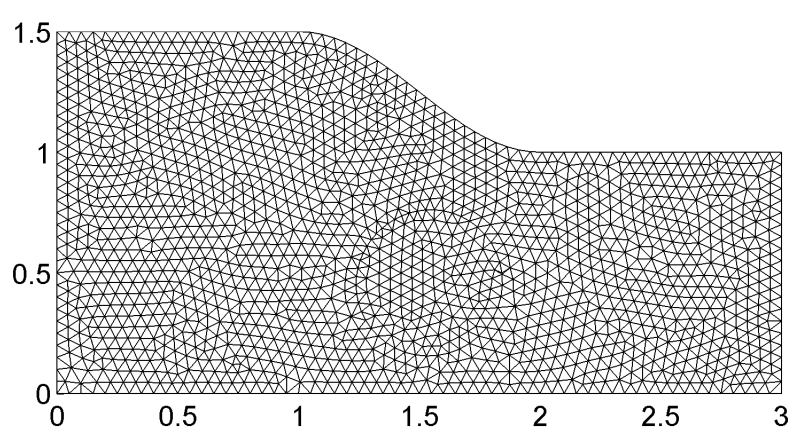

(a)

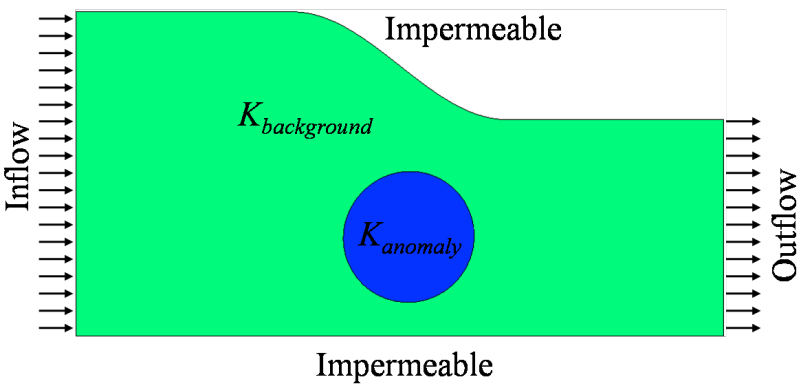

(b)

Figure 1. (a) Domain discretization, (b) geometry model and boundary conditions.

Irregular geometry and variation in permeability distribution models are used. Figure 1 above is the model that we investigated in this modeling. Fluid inflow presented on the left boundary of the domain and fluid outflow presented on the right boundary of the domain with equal volume flow rate. While at the top boundary and bottom boundary of the domain is an impermeable boundary where there is no fluid inflow or outflow. According to this model, the type of boundary conditions need to be imposed are Neumann boundary conditions for inflow, outflow and impermeable boundary. In general form, Neumann boundary condition can be written as

$$
K \frac{\partial \phi}{\partial n}=q
$$

The value of $q$ in equation above obtained from the value of fluid flow velocity in the normal direction to the domain boundary which is positive for inflow and negative for outflow. Neumann boundary condition used in this modeling is $q=1$ $\mathrm{m} / \mathrm{s}$ for the the inflow, $q=-1.5 \mathrm{~m} / \mathrm{s}$ for the outflow, and $q=0 \mathrm{~m} / \mathrm{s}$ for the impermeable boundary. In addition to Neumann boundary condition above, Dirichlet boundary condition is also used at a point in the modeling domain which is placed on the coordinate $(0,0)$ of domain with $\varphi=0$. The Dirichlet boundary condition acting as reference for fluid 
head solutions and also ensuring a unique solution for the problems.

In this paper, the solution of fluid head and velocity of fluid flow calculated for three models. All three models are distinguished by the value of anomalous permeability $\left(K_{\text {anomaly }}\right)$. Model 1 is the model with $K_{\text {anomaly }}=K_{\text {background }}$. Model 2 is the model with $K_{\text {anomaly }}=0.1 K_{\text {background. }}$. Model 3 is the model with $K_{\text {anomaly }}=10 K_{\text {background }}$. Geometry and domain boundaries are same for all three models as shown in Figure 1. Value of $K_{\text {background }}$ used in all three models is $1 \mathrm{~m} / \mathrm{s}$.

The finite element method used to generate fluid head solution in the modeling domain. The obtained solution of the fluid head is relative fluid head to the fluid head at reference point $(0,0)$. Solution of fluid flow velocity is obtained by calculating the gradient of the fluid head numerically using equation (2).

\section{RESULTS AND DISCUSSION}

Results of 2D modeling of steady flow in a porous medium are distribution of fluid head and fluid flow velocity in the modeling domain. The following figures are solutions of fluid head obtained for all three models.

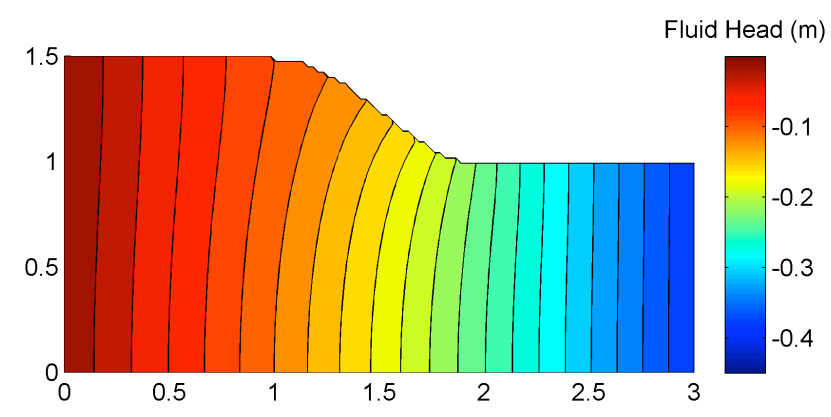

Figure 2. Fluid head contour plot of model $1\left(K_{\text {anomaly }}=\right.$ $K_{\text {background }}$ )

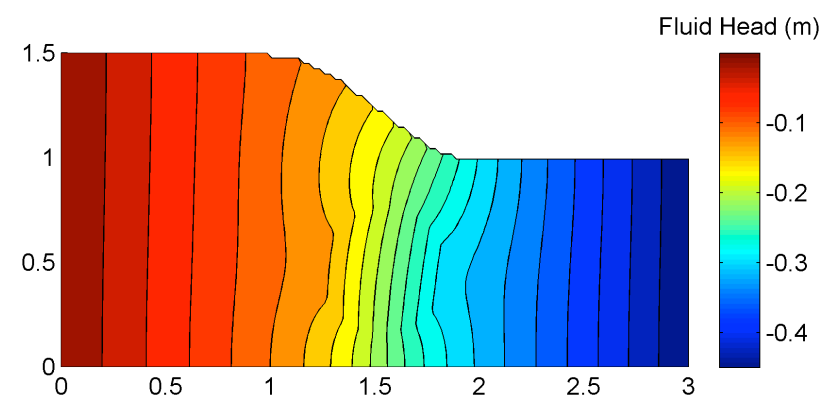

Figure 3. Fluid Fluid head contour plot of model $2\left(K_{\text {anomaly }}=\right.$ $\left.0.1 K_{\text {background }}\right)$

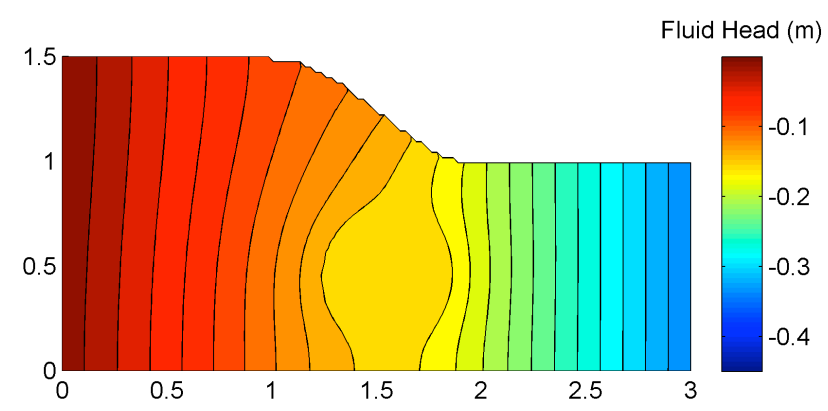

Figure 4. Fluid head contour plot of model $3\left(K_{\text {anomaly }}=\right.$ $10 K_{\text {background) }}$

The following figures are solutions of fluid flow velocity and streamline obtained for all three models.

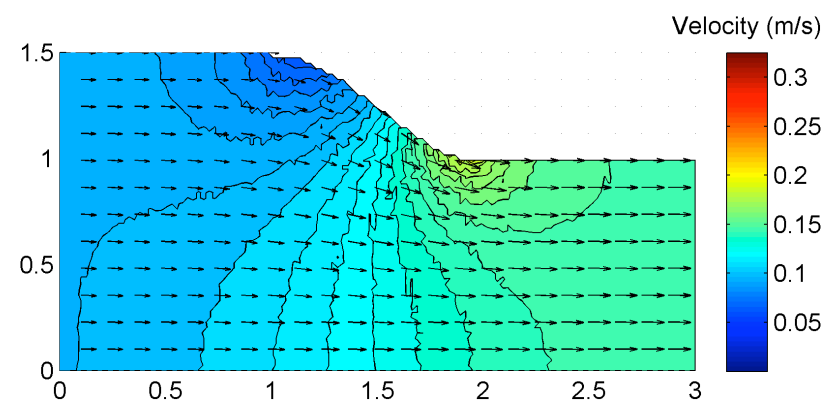

Figure 5. Fluid flow velocity contour plot and quiver plot of model 1 ( $\left.K_{\text {anomaly }}=K_{\text {background }}\right)$

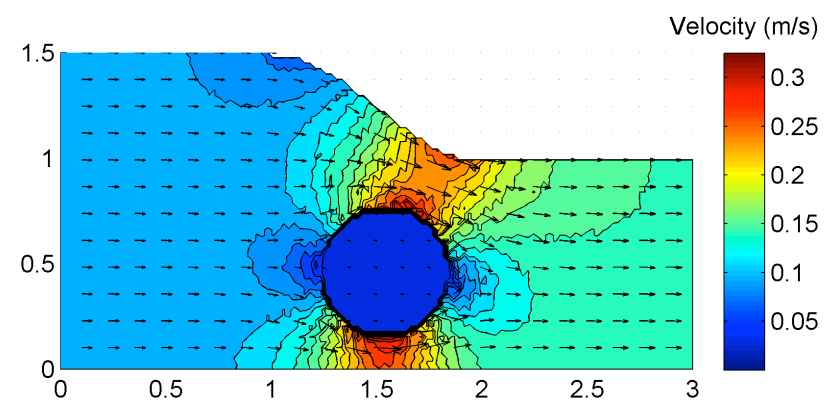

Figure 6. Fluid flow velocity contour plot and quiver plot of model $2\left(K_{\text {anomaly }}=0.1 K_{\text {background }}\right)$

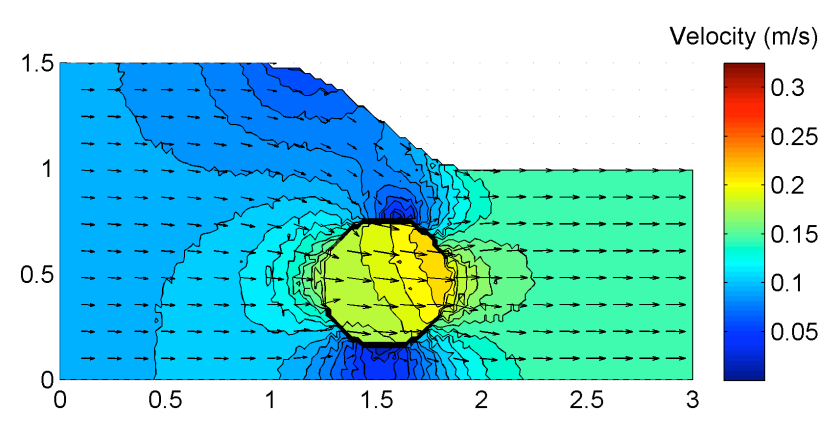

Figure 7. Fluid flow velocity contour plot and quiver plot of model $3\left(K_{\text {anomaly }}=10 K_{\text {background }}\right)$ 


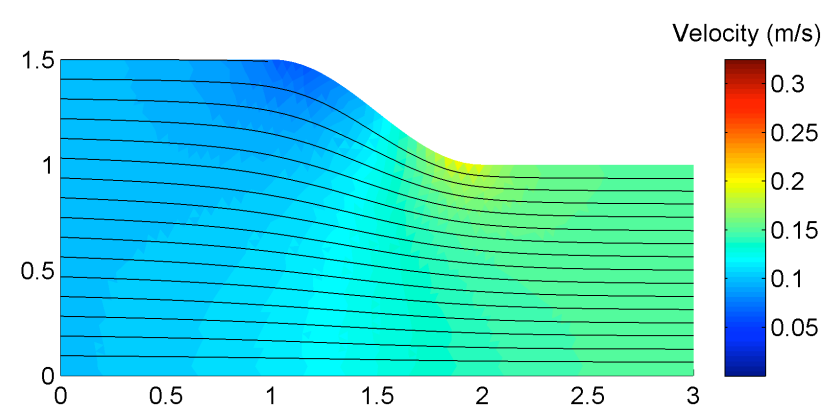

Figure 8. Fluid streamline plot of model $1\left(K_{\text {anomaly }}=K_{\text {background }}\right)$

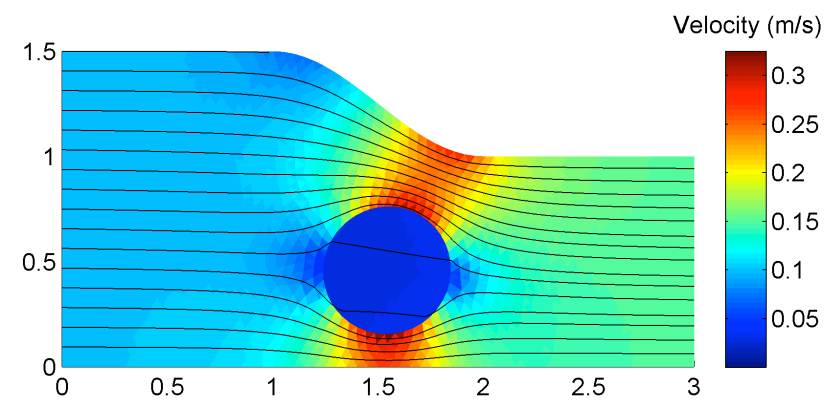

Figure 9. Fluid streamline plot of model $2\left(K_{\text {anomaly }}=\right.$ $\left.0.1 K_{\text {background }}\right)$

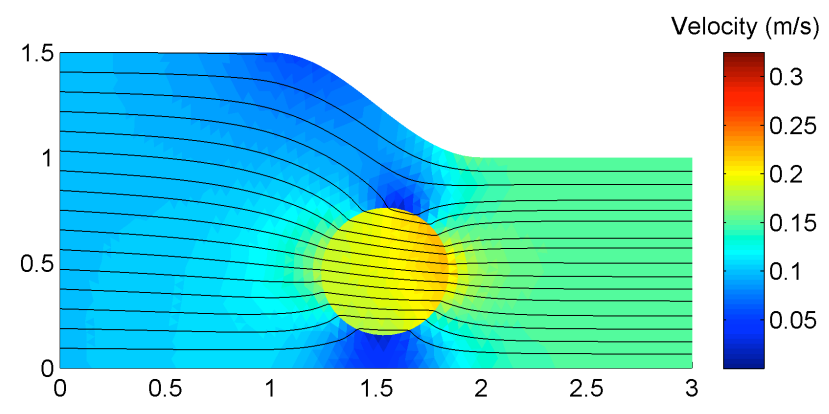

Figure 10. Fluid streamline plot of model $3\left(K_{\text {anomaly }}=\right.$ $10 K_{\text {background }}$ )

Fluid head solutions shown in Figure 2, 3, and 4 for all three models. The presence of permeability contrast anomaly gives a distortion in the results of the fluid head contour solution. In the case of anomalous permeability smaller than the background permeability, fluid head contour lines have a tendency to approach the anomalous area. In the case of anomalous permeability larger than the background permeability, fluid head contour lines have a tendency to evade the anomalous area.

Figure 5, 6, and 7 are the contours of fluid flow velocity. Fluid flow velocity obtained by calculating the resultant of fluid head gradient in $x$ and $y$ direction. Fluid flow direction indicated by the direction of quiver plot on the figures. This plot show the movement of fluid particles within the modeling domain. Smaller fluid flow velocity observed in the anomalous area where anomalous permeabilty smaller than background permeability. Acceleration of the fluid flow observed at the top and bottom outside of anomalous area, as well as a slowdown when entering and exiting the area. As for greater anomalous permeability, otherwise behaviour observed. Fluid flow velocity contour obtained from the modeling using the linear triangular element is less smooth.

Based on the velocity solution, streamline plot for all three models also presented as shown in Figure 8, 9, and 10. The streamline plot show how the existence of anomalous permeability affect the fluid streamline. In the case of anomalous permeability smaller than the background permeability, most of the fluid flow passing through the area around the anomalous area, and only small fraction entering anomalous area. As for greater anomalous permeability, most of the flow entering the anomalous area and only a small portion that passes through the area around the anomalous area.

\section{CONCLUSION}

Two-dimensional steady flow modeling of ideal fluid has been performed using finite element method to all three models which have irregular geometry and variation in permeability distribution. The solutions obtained in form of fluid head and fluid flow velocity distribution in the modeling domain. The Influences of permeability contrast anomalies have been modeled and compared. In this modeling, Fluid flow velocity contour which is calculated from the gradient of the fluid head for the linear triangular element is less smooth. The use of higher order elements could be a solution to overcome this problem. Two-dimensional modeling of steady flow in a porous medium could be applied to groundwater flow pattern modeling for porous aquifer, self potential modeling due to groundwater flow, as material to develop advance simulation in geothermal energy and complex fluid flow simulation.

\section{ACKNOWLEDGMENT}

The first author aknowledges supports from the Indonesia Endowment Fund for Education (LPDP).

\section{REFERENCES}

[1] Darcy, H. P. G., 1856, Les Fontaines Publiques de la Ville de Dijon, Victor Dalmont, Paris.

[2] Dou, C., Woldt, W., Dahab, M. and Bogardi, I. (1997), Transient Ground-Water Flow 
Simulation Using a Fuzzy Set Approach. Groundwater, 35: 205-215.

[3] David Keith Todd, Larry W. Mays, "Groundwater Hydrology", 3rd Edition, John Wiley \& Sons, USA, 2005.
[4] Daryl L. Logan, "A First Course in the Finite Element Method", Thomsons, Canada, 2007.

[5] Anastasis C. Polycarpu, "An Introduction to the Finite Element Method in Electromagnetism", Morgan \& Claypool Publisher, USA, 2006. 